incident involving toxic chemicals. The long table of health hazard summaries for industrial and occupational chemicals may well be useful under such circumstances, as may the list of suspected human carcinogens. These have less relevance for the practising Accident and Emergency (A\&E) physician but this section is brief and worth keeping within the clinical manual as a reference source.

The only small disappointment was that a book described as the International Edition' should detail lists of poisons centres and methods of labelling toxic compounds during transport, relating only to the United States. However, despite that, it is an extremely useful and easily read, well set out source of information that should be in every A\&E department and probably in the pocket of anyone involved in the emergency management of a poisoned patient.

\title{
M. WATERS
}

Stockport Infirmary

Stockport

Cheshire

\section{Practical Fracture Treatment (2nd Edition)}

By R. MCRAE

Churchill Livingstone, Edinburgh. 1989, £22.95, 328pp.

The successful format of annotated diagrams and self-test questions and answers has been reproduced and extended for the second edition of this very useful book.

The first part is suitable for under- or post-graduate students with a minimal prior knowledge of orthopaedics. It sets out the basic pathology of fractures, principles of diagnosis and management, and recognition of complications.

The second, and longer part, considers bony injuries region by region. Of particular value to the accident and emergency (A\&E) doctor and aspiring orthopaedic surgeon is the emphasis that has been placed on the mechanism by which injury may be produced. Line drawings convey the effects of applying percussive and rotational forces to single bones and joints and more complex 'ring' structures. The quality of reproduction of $X$-rays to illustrate this is excellent. Drawings are then used to demonstrate the method of reduction for displaced fractures. There are a considerable number of self-test questions which reinforce earlier teaching.

The author stresses in the preface that he does not seek to be dogmatic with respect to treatment, but to present a range of accepted treatment options.

Practical Fracture Treatment is successful not only as a quick reference guide for the junior doctor, but also as a manual which explains how the bony skeleton sustains and responds to injury. It should be in every A\&E department and on the bookshelves of anyone involved with treating injured patients.

M. WATERS

Stockport Infirmary

Stockport

Cheshire 